Method: We asked those children (males and females aged between 10 and 15 years) 'what we mean by Mediterranean diet?'. Then we proposed a scenario: 'you are the director of a film stage in which a family, mother and father with their sons are eating at home; they live in fifties at the seaside and the father is a fisherman; now you put them around the table of their kitchen and write what we have to put in to realize this scene; if possible, explain also what kind of foods there were in pantry and in refrigerator'.

Results: Seventy-nine children: nobody answered in a correct way to the question regarding Md. Only twenty- eight (about 35\%) put on the kitchen's table something according to Md: fish, bread, season vegetables, fruit and in some cases a simple dish of spaghetti with tomato sauce, water and red wine and olive oil. The others really only tried to put on that table all kinds of possible foods they know.

Conclusions: Only few children recognize Md: they do not know either blue fish and its properties or vegetables and fruits. They do not think about simple bread and olives or pressed cheese at all. We recognize there is great need of education with practical examples, trying to teach nutritional properties of food in a more complete way to young people.

\title{
65 - Front-of-pack logo v. Guideline Daily Amounts: what's the better tool to promote suitable choices of packaged products in children according to health professionals?
}

\author{
TD Spagnoli ${ }^{1}, M$ Caputo $^{2}$, R Magliola ${ }^{2}, M$ Audenino ${ }^{2}$ and L Cesari ${ }^{1}$ \\ ${ }^{1}$ ASL Turin 3 Service of Nutrition and Food Hygiene, Collegno, Turin, Italy: 2Piedmont Regional Department for \\ Health Promotion, Turin, Italy
}

Introduction: A lot of tools, such as Front-of-Pack nutritional Logos and Guideline Daily Amounts (GDA), are avaiable on labels in several countries to help consumers in choosing packaged foods. We assessed, according to health professionals, which tool could better help parents in the choice of snacks to prevent overweight in children.

Method: We performed focus groups on health professionals of the Departments of Preventive Nutrition (SIAN Services) in the whole Piedmont (an Italian region) and on paediatricians of a randomized district in Piedmont (Pinerolo). We compared GDA and logos placed on the labels from the United Kingdom (traffic-light), Finland (heart), Sweden (key), New Zealand (thick), Canada (health check), France (nutritional cursor) and The Netherlands (health choice).

Results: Forty-eight out of fifty-one health professionals (94\%) were interviewed: thirty-four SIAN workers (11/13 regional services were represented) and fourteen paediatricians. In all $60 \%$ of interviewed professionals (29/48) chose the traffic-light, $21 \%(10 / 48)$ the French-cursor, 19\% (9/48) another logo; none chose the GDA. The prevalent explanations were clearness and understanding of the logo: it seemed more effective in communication than GDA because it was simpler and it was a picture, so could be easily seen and understood by everyone, even by people who have reading troubles. Moreover, among the different logos, the traffic-light seemed the most effective because it is universally understandable, even by children.

Conclusions: In Italy, the Confederation of Food-Industries (Federalimentare) promotes the spreading of GDA on packaged foods. However, data from health professionals are in agreement with results from other studies, suggesting that the GDA are less efficient tools than the logo.

Funding: Research relating to this abstract was selffunded by Piedmont Region and ASL Turin 3

Thanks to D Lo Bartolo, L Bioletti, S Spagna, S Ropolo, A Cosola, O Maganuco, G Gibilisco (ASL TO 3), and to all the health professionals interviewed.

\section{6 - Health effects of lifestyle interventions in obese children and adolescents study (HELIOS) randomised controlled trial}

\author{
S Makkes ${ }^{1}$, J Halberstadt ${ }^{1}, \mathrm{CM}$ Renders ${ }^{1}, \mathrm{JE} \mathrm{Bosmans}^{1}, \mathrm{OH}$ van der Baan-Slootweg ${ }^{2}$ and \\ JC Seidell ${ }^{1}$ \\ 'Department of Health Sciences and the EMGO Institute for Health and Care Research, VU University Amsterdam, \\ The Netherlands: ${ }^{2}$ Koepel Behandelcentra Chronisch Zieken (KBCZ), Heideheuvel, Hilversum, The Netherlands
}


Background: The prevalence of obesity among children is increasing in the Netherlands. Intensive treatment for severe obesity in children is required, but evidence-based costeffective options are not yet available.

Aim: To compare the cost-effectiveness of two intensive one-year inpatient interventions and outpatient treatment in severely obese children and adolescents. The two inpatient interventions are different with respect to the length of the hospitalization period.

Design: A randomized clinical trial with three study arms.

Setting and subjects: Eighty children aged 8-12 years and adolescents aged 12-18 years admitted to the KBCZ in Hilversum, the Netherlands, with therapy resistant severe obesity (SDS-BMI $>3 \cdot 0$ or SDS-BMI $>2 \cdot 3$ with obesity-related comorbidity).

Interventions: Group A receives inpatient treatment for 6 months during weekdays. Group B receives inpatient treatment for 2 months, followed by biweekly hospital admissions for $2 \mathrm{~d}$ during 4 months. In the second half year there are six sessions of $2 \mathrm{~d}$ aimed at reinforcement of learned behaviour in both groups. Both intervention programs are intensive lifestyle programs with emphasis on nutrition, exercise and cognitive behavioural therapy. In both programs active participation of the parents is required. Group C receives usual care for a year after which they are randomized to treatment A or B.

Preliminary results: Mean baseline SDS-BMI for adolescents was 3.4 and 3.3 for children. High triglycerides were observed in $6.7 \%$ and $5 \%$ of adolescents and children, respectively. Low HDL-cholesterol was observed in $56.7 \%$ and $60 \%$ of adolescents and children. Hypertension was more prevalent in adolescents $(53 \cdot 6 \%$ v. $10 \cdot 5 \%, P<0 \cdot 01)$. Intensive treatment is warranted.

\title{
67 - 'http://HANCPtool.org' as the first step to school menu reformulations
}

\author{
A Colom ${ }^{1}$, M Autonell ${ }^{1}$, M Buades ${ }^{1}$, E Cabeza ${ }^{1}$, E Ferragut ${ }^{1}$, P Palou ${ }^{1}$, Y Vega ${ }^{1}$, \\ $M$ Monino $^{2}$, M Colomer $^{2}$, A Terrassa $^{2}$ and FA Colom ${ }^{2}$ \\ ${ }^{1}$ Balearic Islands Public Health Department, Spain: ${ }^{2}$ Balearic Dieticians and Nutritionists' Official Association, Spain
}

Introduction: Diet-related diseases are becoming growing epidemics and are major contributors to the leading causes of childhood unhealthiness in Europe.

Rationale: Obesity and weight-problem prevention by stimulating food reformulation to modify school diets by reducing the contents of saturated fatty acids (SFA), sodium (salt) and free sugars (extrinsic sugars).

Method: Development of a new tool based on the classic HACCP (Hazard Analysis and Critical Control Points) to translate scientific knowledge into operational terms:

- 'FNO': Food Nutritional Objective: the maximum concentration of a nutritional risk.
- 'NPC': Nutritional Performance Criteria: the desired concentration reduction of a nutritional risk.

- 'WONRAC': Workplace Nutritional Risk Assessment and Control: points where something is added or a process made that can alter the contents monitored by Google analytics to bring about an awareness of the Health Impact Assessment (HIA).

Results: The newly developed tool, HANCPtool (Hazard Analysis Nutritional Control Points) is presented in the web2.0 area of http://www.foodprofit.org

Conclusions: This approach facilitates the measurement of nutrients in order to understand the leading problem, improve them via reformulation and predict the HIA consequences of school menus.

\section{8 - EMPOWER - empowering parents to prevent obesity at weaning - exploratory research}

\author{
R Lang' ', Barlow', S Whitlock', D Kaklamanou' ${ }^{2}$ S Hanson ${ }^{3}$, K Sylvester ${ }^{4}$ and MC Rudolf ${ }^{2,3}$ \\ 'University of Warwick, Warwick Medical School, Gibbet Hill, Coventry, UK: ${ }^{2}$ University of Leeds, Clarendon \\ Wing, Leeds General Infirmary, Belmont Grove, Leeds, UK: ${ }^{3}$ Leeds Primary Care Trust, Leeds, UK: ${ }^{4}$ Heart of \\ Birmingham Primary Care Trust, Birmingham, UK
}

